\title{
Electrical Conductivity Measurement on Metallic Materials With a Cylindrical Resonator
}

Dong, Yunfeng; Jellesen, Morten Stendahl; Christiansen, Rune Juul; Høvelskov, Jesper; Sundgren, Jörgen; Jakobsen, Kaj Bjarne

Published in:

IEEE Transactions on Electromagnetic Compatibility

Link to article, DOI:

10.1109/TEMC.2021.3070082

Publication date:

2021

Document Version

Peer reviewed version

Link back to DTU Orbit

Citation (APA):

Dong, Y., Jellesen, M. S., Christiansen, R. J., Høvelskov, J., Sundgren, J., \& Jakobsen, K. B. (2021). Electrical Conductivity Measurement on Metallic Materials With a Cylindrical Resonator. IEEE Transactions on

Electromagnetic Compatibility, 63(6), 1875 - 1883. https://doi.org/10.1109/TEMC.2021.3070082

\section{General rights}

Copyright and moral rights for the publications made accessible in the public portal are retained by the authors and/or other copyright owners and it is a condition of accessing publications that users recognise and abide by the legal requirements associated with these rights.

- Users may download and print one copy of any publication from the public portal for the purpose of private study or research.

- You may not further distribute the material or use it for any profit-making activity or commercial gain

- You may freely distribute the URL identifying the publication in the public portal 


\title{
Electrical Conductivity Measurement on Metallic Materials With a Cylindrical Resonator
}

\author{
Yunfeng Dong 로 Member, IEEE, Morten Stendahl Jellesen, Rune Juul Christiansen, Jesper Høvelskov, \\ Jörgen Sundgren, and Kaj Bjarne Jakobsen
}

\begin{abstract}
This article presents a novel method for measuring the electrical conductivity of metallic materials using a cylindrical resonator. The theory and the process of measuring the electrical conductivity are explained. The cylindrical resonator is designed and proved using full-wave electromagnetic simulations. A prototype operating at $10.5 \mathrm{GHz}$ is fabricated, and the scattering parameters are measured. The quality factor of the cylindrical resonator is calculated at the first step from the measured scattering parameters, and the electrical conductivity of the metallic material is derived using Maxwell's equations. By replacing the top cover of the cylindrical resonator with different materials or metallic sheets, the electrical conductivities can be measured easily. For validating the method, three top covers and six metallic sheets are fabricated and tested. The measured electrical conductivities are compared with other published results and typical values. Besides, by adjusting the operating frequency of the cylindrical resonator, the proposed method can be applied to determine the electrical conductivity at specific frequencies.
\end{abstract}

Index Terms-Cylindrical resonator, electrical conductivity, Maxwell's equations, quality factor, scattering parameters, surface resistivity.

\section{INTRODUCTION}

$\mathbf{E}$ LECTRICAL conductivity $(\sigma)$ of a material, referring to the inverse of electrical resistivity $(R)$, is a fundamental property that indicates the ability of conducting electric current. Though the typical values of the electrical conductivities for metallic materials such as copper, aluminum, brass, and steel have been widely used for decades, they are mainly characterized by direct current (DC) using Ohm's law, whereas, for customized materials or applications operating at microwave frequencies, the electrical conductivity varies and individual characterization becomes necessary [1]-[3]. In addition, due to skin effect,

Manuscript received November 6, 2020; revised January 26, 2021; accepted March 27, 2021. This work was supported by the Eurostars-funded project Micro-Shield under Contract E12496. (Corresponding author: Yunfeng Dong.)

Yunfeng Dong and Kaj Bjarne Jakobsen are with the Electromagnetic Systems Group, Department of Electrical Engineering, Technical University of Denmark, 2800 Kongens Lyngby, Denmark (e-mail: yundon@elektro.dtu.dk; kbj@elektro.dtu.dk).

Morten Stendahl Jellesen is with Materials and Surface Engineering Group, Department of Mechanical Engineering, Technical University of Denmark, 2800 Kongens Lyngby, Denmark (e-mail: msj@mek.dtu.dk).

Rune Juul Christiansen and Jesper Høvelskov are with AH Metal Solutions A/S, 3450 Lillerød, Denmark (e-mail: rch@ah-dk.dk; jh@ah-dk.dk).

Jörgen Sundgren is with Lysekil Plating Technology AB, 45338 Lysekil, Sweden (e-mail: jorgen.sundgren@lptech.se).

Color versions of one or more figures in this article are available at https://doi.org/10.1109/TEMC.2021.3070082.

Digital Object Identifier 10.1109/TEMC.2021.3070082

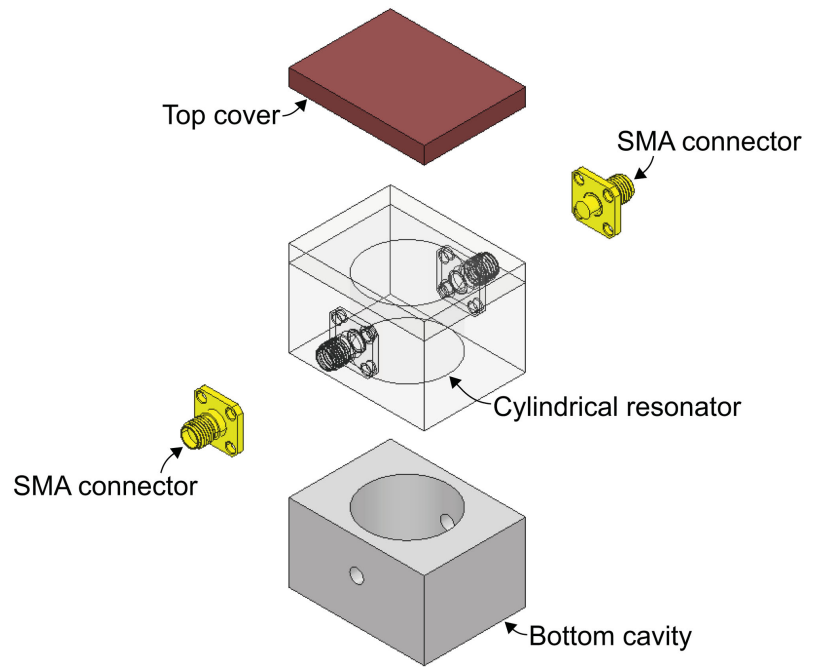

Fig. 1. Overview of the proposed $\mathrm{TE}_{111}$ mode cylindrical resonator.

metallic sheets with thin cladding layers, engineered composites, and alloys may exhibit different properties [4]-[8]. As is reported in [1], a $\mathrm{TE}_{011}$ mode cavity resonator is used for characterizing the electrical conductivities of metallic sheets. According to [9]-[12], electrical conductivities can be characterized by eddy current using Faraday's law of induction. Other methods for measuring the electrical conductivity, permittivity $(\varepsilon)$, and permeability $(\mu)$ are also published in the literature [13]-[17]. However, these methods either rely on complex structures or consist of dedicated components such as dielectric resonator, spacer, loop feed, magnet, and coil. Fig. 1 shows the overview of the proposed cylindrical resonator. In the closed cavity, electric waves reflect back and forth between the conductor walls forming a standing wave of $\mathrm{TE}_{111}$ mode. By replacing the top cover with different materials or metallic sheets with thin cladding layers, the electrical conductivities can be measured at the resonant frequency $(f)$. While having a simple structure, the cylindrical resonator also provides a low-cost and easy way of measuring the electrical conductivity of metallic materials at microwave frequencies. Furthermore, by scaling the cavity and replacing SMA connectors with waveguides, the proposed method can be potentially used for characterizing electrical conductivities at millimeter-wave and terahertz frequencies.

In this article, a novel method for characterizing the electrical conductivity of metallic materials at microwave frequencies is addressed. The cylindrical resonator is designed and proved 


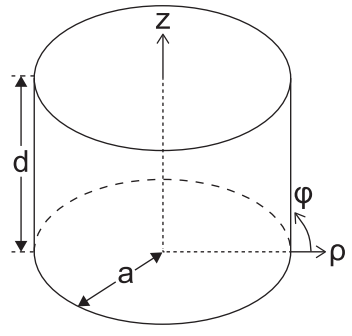

Fig. 2. Air-filled cylindrical resonator ( $\rho \varphi z$-coordinate system).

using full-wave electromagnetic simulations in ansys electronics desktop (EDT, formerly known as HFSS). A prototype is fabricated, and different metallic materials are tested. By measuring the scattering parameters of the proposed $\mathrm{TE}_{111}$ mode cylindrical resonator, the quality factor $(Q)$ can be calculated from the measured half-power (3-dB) bandwidth. In the meanwhile, the quality factor of the cylindrical resonator is determined by the angular frequency $(\omega)$, the average stored energy $(W)$, and the power loss $\left(P_{1}\right)$ due to using nonperfect metallic materials. As a result, the electrical conductivity of the conductor walls of the cylindrical resonator can be derived by the measured quality factor using Maxwell's equations.

The rest of this article is organized as follows. In Section II, the theory of deriving the electrical conductivities of the conductor walls from the quality factor of a cylindrical resonator by using Maxwell's equations is introduced in detail. In Section III, the fabrication of the proposed cylindrical resonator operating at $10.5 \mathrm{GHz}$ is presented. The assembly structure and the way of measuring metallic materials are illustrated. In Section IV, the experimental results are presented and compared with the typical values as well as published results. In Section V, the accuracy and the uncertainties of the characterized electrical conductivities are discussed. Finally, Section VI concludes this article.

\section{THEORY}

\section{A. Electrical Conductivity}

As shown in Fig. 2, the proposed cylindrical resonator is a hollow closed conductor, where the electromagnetic waves form a standing wave of $\mathrm{TE}_{111}$ mode inside the cavity at the resonant frequency. When being filled with air, the relative permeability $\left(\mu_{\mathrm{r}}\right)$ and the relative permittivity $\left(\varepsilon_{\mathrm{r}}\right)$ of the cylindrical resonator are assumed to be 1 , and the variation within frequency is neglected in the calculation.

In this case, the radius and the height of the air-filled cylindrical resonator are represented by $a$ and $d$, respectively. Based on Maxwell's equations, the electric field $(E)$ and magnetic field $(H)$ components of the $\mathrm{TE}_{n m l}$ mode in the $\rho \varphi z$-coordinate system can be written as

$$
\begin{aligned}
& E_{\rho}=\frac{j k \eta a^{2} n H_{0}}{P_{n m}^{\prime 2} \rho} \mathrm{J}_{\mathrm{n}}\left(\frac{P_{n m}^{\prime} \rho}{a}\right) \sin (n \varphi) \sin \left(\frac{l \pi z}{d}\right) \\
& E_{\varphi}=\frac{j k \eta a H_{0}}{P_{n m}^{\prime}} \mathrm{J}_{\mathrm{n}}^{\prime}\left(\frac{P_{n m}^{\prime} \rho}{a}\right) \cos (n \varphi) \sin \left(\frac{l \pi z}{d}\right) \\
& E_{z}=0
\end{aligned}
$$

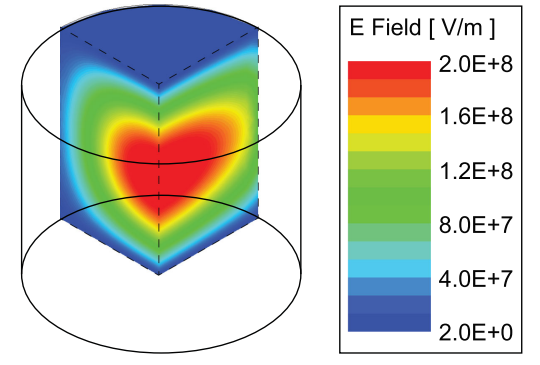

Fig. 3. Electric field distribution of a $\mathrm{TE}_{111}$ mode cylindrical resonator.

$$
\begin{aligned}
& H_{\rho}=\frac{\beta a H_{0}}{P_{n m}^{\prime}} \mathrm{J}_{\mathrm{n}}^{\prime}\left(\frac{P_{n m}^{\prime} \rho}{a}\right) \cos (n \varphi) \cos \left(\frac{l \pi z}{d}\right) \\
& H_{\varphi}=\frac{-\beta a^{2} n H_{0}}{P_{n m}^{\prime 2} \rho} \mathrm{J}_{\mathrm{n}}\left(\frac{P_{n m}^{\prime} \rho}{a}\right) \sin (n \varphi) \cos \left(\frac{l \pi z}{d}\right) \\
& H_{z}=H_{0} \mathrm{~J}_{\mathrm{n}}\left(\frac{P_{n m}^{\prime} \rho}{a}\right) \cos (n \varphi) \sin \left(\frac{l \pi z}{d}\right)
\end{aligned}
$$

where $J_{n}$ is the first kind of Bessel function, $J_{n}^{\prime}$ represents the derivative of $\mathrm{J}_{\mathrm{n}}$, and $P_{n m}^{\prime}$ refers to the $m$ th root of $\mathrm{J}_{\mathrm{n}}^{\prime}$. Other parameters, including the propagation constant $(\beta)$, the wave number $(k)$, the intrinsic impedance $(\eta)$, and the arbitrary amplitude $\left(H_{0}\right)$, can be calculated as

$$
\begin{aligned}
& \beta=\sqrt{k^{2}-\left(\frac{P_{n m}^{\prime}}{a}\right)^{2}} \\
& k=2 \pi f \sqrt{\mu \varepsilon}=2 \pi f \sqrt{\mu_{0} \mu_{\mathrm{r}} \varepsilon_{0} \varepsilon_{\mathrm{r}}} \\
& \eta=\sqrt{\frac{\mu}{\varepsilon}}=\sqrt{\frac{\mu_{0} \mu_{\mathrm{r}}}{\varepsilon_{0} \varepsilon_{\mathrm{r}}}} \\
& H_{0}=-2 j A^{+}
\end{aligned}
$$

where $\mu_{0}$ and $\varepsilon_{0}$ represent the permeability and permittivity of free space, respectively, and $A^{+}$is the arbitrary amplitudes of the forward traveling wave.

For the proposed $\mathrm{TE}_{111}$ mode cylindrical resonator, the resonant frequency $\left(f_{111}\right)$ can be expressed as

$$
f_{111}=\frac{c}{2 \pi \sqrt{\mu_{\mathrm{r}} \varepsilon_{\mathrm{r}}}} \sqrt{\left(\frac{P_{11}^{\prime}}{a}\right)^{2}+\left(\frac{\pi}{d}\right)^{2}}
$$

where $P_{11}^{\prime}=1.841, c$ represents the speed of light in free space, and $\mu_{\mathrm{r}}=\varepsilon_{\mathrm{r}}=1$ is satisfied because of the approximation for the air-filled cavity. Fig. 3 illustrates the electric field distribution of the proposed cylindrical resonator, which is cut into a quarter showing the $\mathrm{TE}_{111}$ mode inside the cavity. When resonance occurs, the average stored electric energy $\left(W_{\mathrm{e}}\right)$ equals the average stored magnetic energy $\left(W_{\mathrm{h}}\right)$. As a result, the total stored energy $(W)$ of a $\mathrm{TE}_{n m l}$ mode cylindrical resonator can be written in terms of a volume integral using the electric field components introduced in (1) as

$$
\begin{aligned}
& W=W_{\mathrm{e}}+W_{\mathrm{h}}=2 W_{\mathrm{e}} \\
& W=\frac{\varepsilon}{2} \int_{z=0}^{d} \int_{\varphi=0}^{2 \pi} \int_{\rho=0}^{a}\left(\left|E_{\rho}\right|^{2}+\left|E_{\varphi}\right|^{2}\right) \rho \mathrm{d} \rho \mathrm{d} \varphi \mathrm{d} z
\end{aligned}
$$




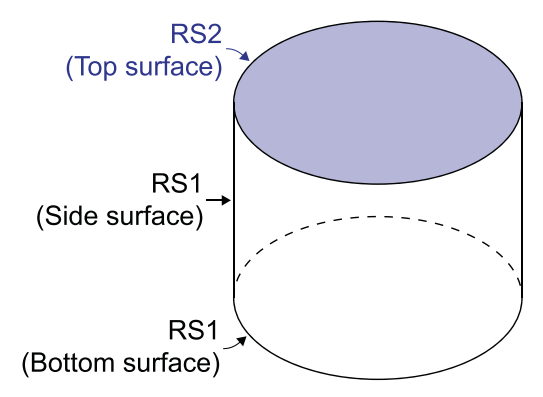

Fig. 4. Cylindrical resonator made by nonperfect metallic materials.

$$
\begin{aligned}
&=\frac{\varepsilon k^{2} \eta^{2} a^{2} \pi d H_{0}^{2}}{4\left(P_{n m}^{\prime}\right)^{2}} \int_{\rho=0}^{a}\left[\mathrm{~J}_{\mathrm{n}}^{\prime 2}\left(\frac{P_{n m}^{\prime} \rho}{a}\right)\right. \\
&\left.+\left(\frac{n a}{P_{n m}^{\prime} \rho}\right)^{2} \mathrm{~J}_{\mathrm{n}}^{2}\left(\frac{P_{n m}^{\prime} \rho}{a}\right)\right] \rho \mathrm{d} \rho \\
& W=\frac{\varepsilon k^{2} \eta^{2} a^{4} H_{0}^{2} \pi d}{8 P_{n m}^{\prime 2}}\left[1-\left(\frac{n}{P_{n m}^{\prime}}\right)^{2}\right] \mathrm{J}_{\mathrm{n}}^{2}\left(P_{n m}^{\prime}\right) .
\end{aligned}
$$

If the conductor wall of a resonator is made by a perfect electric conductor (PEC), there will not be any conductor loss due to infinite electrical conductivity. That means the corresponding power loss also becomes zero and results in an infinite quality factor. As shown in Fig. 4, nonperfect metallic materials are used for the cylindrical resonator in reality. In this case, due to different metallic materials, the side and bottom surfaces have a surface resistivity of $R_{\mathrm{S} 1}$, while it is $R_{\mathrm{S} 2}$ for the top surface. Since the surface resistivity is the inverse of electrical conductivity, it can be written as

$$
R_{\mathrm{S} 1,2}=\sqrt{\frac{2 \pi f \mu}{2 \sigma_{1,2}}}
$$

where $\sigma_{1,2}$ represents the electrical conductivities of the corresponding metallic materials. In other words, once the surface resistivity is known, the electrical conductivity can be found. For calculating the power loss of the cylindrical resonator, the magnetic field components introduced in (1) also need to be rewritten for each surface as

Side surface:

$$
\begin{aligned}
& H_{\varphi,(\rho=a)}=\frac{-\beta a n H_{0}}{P_{n m}^{\prime 2}} \mathrm{~J}_{\mathrm{n}}\left(P_{n m}^{\prime}\right) \sin (n \varphi) \cos \left(\frac{l \pi z}{d}\right) \\
& H_{z,(\rho=a)}=H_{0} \mathrm{~J}_{\mathrm{n}}\left(P_{n m}^{\prime}\right) \cos (n \varphi) \sin \left(\frac{l \pi z}{d}\right)
\end{aligned}
$$

Bottom surface:

$$
\begin{aligned}
& H_{\rho,(z=0)}=\frac{\beta a H_{0}}{P_{n m}^{\prime}} \mathrm{J}_{\mathrm{n}}^{\prime}\left(\frac{P_{n m}^{\prime} \rho}{a}\right) \cos (n \varphi) \\
& H_{\varphi,(z=0)}=\frac{-\beta a^{2} n H_{0}}{P_{n m}^{\prime 2} \rho} \mathrm{J}_{\mathrm{n}}\left(\frac{P_{n m}^{\prime} \rho}{a}\right) \sin (n \varphi)
\end{aligned}
$$

Top surface:

$$
H_{\rho,(z=d)}=\frac{\beta a H_{0}}{P_{n m}^{\prime}} \mathrm{J}_{\mathrm{n}}^{\prime}\left(\frac{P_{n m}^{\prime} \rho}{a}\right) \cos (n \varphi) \cos (l \pi)
$$

$$
H_{\varphi,(z=d)}=\frac{-\beta a^{2} n H_{0}}{P_{n m}^{\prime 2} \rho} \mathrm{J}_{\mathrm{n}}\left(\frac{P_{n m}^{\prime} \rho}{a}\right) \sin (n \varphi) \cos (l \pi) .
$$

As a result, by taking into account the surface resistivities of the corresponding metallic materials, the power loss $\left(P_{1}\right)$ of a $\mathrm{TE}_{n m l}$ mode cylindrical resonator can be expressed in terms of three surface integrals using the magnetic field components introduced in (6) as

$$
\begin{aligned}
& P_{1}= \frac{R_{\mathrm{S} 1}}{2} \int_{z=0}^{d} \int_{\varphi=0}^{2 \pi}\left(\left|H_{\varphi,(\rho=a)}\right|^{2}+\left|H_{z,(\rho=a)}\right|^{2}\right) a \mathrm{~d} \varphi \mathrm{d} z \\
&+\frac{R_{\mathrm{S} 1}}{2} \int_{\varphi=0}^{2 \pi} \int_{\rho=0}^{a}\left(\left|H_{\rho,(z=0)}\right|^{2}+\left|H_{\varphi,(z=0)}\right|^{2}\right) \rho \mathrm{d} \rho \mathrm{d} \varphi \\
&+\frac{R_{\mathrm{S} 2}}{2} \int_{\varphi=0}^{2 \pi} \int_{\rho=0}^{a}\left(\left|H_{\rho,(z=d)}\right|^{2}+\left|H_{\varphi,(z=d)}\right|^{2}\right) \rho \mathrm{d} \rho \mathrm{d} \varphi \\
&= \frac{R_{\mathrm{S} 1}}{2} \pi H_{0}^{2} \mathrm{~J}_{\mathrm{n}}^{2}\left(P_{n m}^{\prime}\right)\left[\frac{d a}{2}\left(1+\frac{\beta^{2} a^{2} n^{2}}{P_{n m}^{\prime}}\right)\right] \\
&+\frac{R_{\mathrm{S} 1}}{2} \pi H_{0}^{2} \mathrm{~J}_{\mathrm{n}}^{2}\left(P_{n m}^{\prime}\right)\left[\frac{\beta^{2} a^{4}}{2 P_{n m}^{\prime 2}}\left(1-\frac{n^{2}}{P_{n m}^{\prime 2}}\right)\right] \\
&+\frac{R_{\mathrm{S} 2}}{2} \pi H_{0}^{2} \mathrm{~J}_{\mathrm{n}}^{2}\left(P_{n m}^{\prime}\right)\left[\frac{\beta^{2} a^{4}}{2 P_{n m}^{\prime 2}}\left(1-\frac{n^{2}}{P_{n m}^{\prime 2}}\right)\right] \\
& P_{1}= \frac{\pi H_{0}^{2} \mathrm{~J}_{\mathrm{n}}^{2}\left(P_{n m}^{\prime}\right)}{4}\left[R_{\mathrm{S} 1} d a\left(1+\frac{\beta^{2} a^{2} n^{2}}{P_{n m}^{\prime 4}}\right)+\right. \\
&\left.\frac{\left(R_{\mathrm{S} 1}+R_{\mathrm{S} 2}\right) \beta^{2} a^{4}}{P_{n m}^{\prime 2}}\left(1-\frac{n^{2}}{P_{n m}^{\prime 2}}\right)\right] .
\end{aligned}
$$

So far, the total stored energy and the power loss of a $\mathrm{TE}_{n m l}$ mode cylindrical resonator are derived in (4c) and (7b), respectively. The quality factor $(Q)$ can then be written as

$$
\begin{aligned}
Q= & \frac{\omega W}{P_{1}}=\frac{\frac{k}{\sqrt{\mu \varepsilon}} W}{P_{1}} \\
Q= & \frac{k}{\sqrt{\mu \varepsilon}} \frac{\varepsilon k^{2} \eta^{2} a^{4} H_{0}^{2} \pi d}{8 P_{n m}^{\prime 2}}\left[1-\left(\frac{n}{P_{n m}^{\prime}}\right)^{2}\right] \mathrm{J}_{\mathrm{n}}^{2}\left(P_{n m}^{\prime}\right) . \\
& \left\{\frac { \pi H _ { 0 } ^ { 2 } \mathrm { J } _ { \mathrm { n } } ^ { 2 } ( P _ { n m } ^ { \prime } ) } { 4 } \left[R_{\mathrm{S} 1} d a\left(1+\frac{\beta^{2} a^{2} n^{2}}{P_{n m}^{\prime 4}}\right)\right.\right. \\
& \left.\left.+\frac{\left(R_{\mathrm{S} 1}+R_{\mathrm{S} 2}\right) \beta^{2} a^{4}}{P_{n m}^{\prime 2}}\left(1-\frac{n^{2}}{P_{n m}^{\prime 2}}\right)\right]\right\}^{-1}
\end{aligned}
$$

where $R_{\mathrm{S} 1}$ and $R_{\mathrm{S} 2}$, representing the corresponding surface resistivities, are the only unknowns in the equation once the resonant mode and the dimensions of the cylindrical resonator are specified. By simplifying the equation and isolating $R_{\mathrm{S} 2}$, (8b) can be rewritten in terms of $Q$ and $R_{\mathrm{S} 1}$ as

$$
R_{\mathrm{S} 2}=\left[\frac{k^{3} \eta a^{4} d\left(1-\frac{n^{2}}{P_{n m}^{\prime 2}}\right)}{2 P_{n m}^{\prime 2} Q}-R_{\mathrm{S} 1} a d\left(1+\frac{\beta^{2} a^{2} n^{2}}{P_{n m}^{\prime 4}}\right)\right] .
$$$$
\frac{P_{n m}^{\prime 2}}{\beta^{2} a^{4}\left(1-\frac{n^{2}}{P_{n m}^{\prime 2}}\right)}-R_{\mathrm{S} 1}
$$ 
where $Q$ can be calculated from the measured half-power bandwidth, and $R_{\mathrm{S} 1}$ needs to be provided or characterized. Then, the electrical conductivity of the top surface $\left(\sigma_{2}\right)$ can be calculated by rewriting (5) as

$$
\sigma_{2}=\frac{2 \pi f \mu}{2 R_{\mathrm{S} 2}^{2}} .
$$

As shown in Fig. $4, R_{\mathrm{S} 1}$ in (9) represents the surface resistivity of the side and bottom surfaces of the cylindrical resonator. Instead of directly using the typical value or being achieved from the data sheet, it can also be characterized using the proposed method by replacing the metallic materiel of the top surface with the same metallic material as the side and bottom surfaces. In this case, the cylindrical resonator is covered by homogeneous conductor walls with a surface resistivity of $R_{\mathrm{S} 1}$. By substituting $R_{\mathrm{S} 2}$ with $R_{\mathrm{S} 1}$ in (8b) and simplifying the equation, $R_{\mathrm{S} 1}$ can be written as

$$
\begin{aligned}
& R_{\mathrm{S} 1}= \frac{k^{3} \eta a^{4} d\left(1-\frac{n^{2}}{P_{n m}^{\prime 2}}\right)}{4 P_{n m}^{\prime 2} Q_{0}}\left[\frac{\beta^{2} a^{4}}{P_{n m}^{\prime 2}}\left(1-\frac{n^{2}}{P_{n m}^{\prime 2}}\right)\right. \\
&\left.+\frac{a d}{2}\left(1+\frac{\beta^{2} a^{2} n^{2}}{P_{n m}^{\prime 4}}\right)\right]^{-1}
\end{aligned}
$$

where $Q_{0}$ refers to the corresponding quality factor when the top cover of the proposed cylindrical resonator has a surface resistivity of $R_{\mathrm{S} 1}$.

\section{B. Quality Factor}

Though the quality factor of the cylindrical resonator can be calculated from the measured scattering parameters using half-power bandwidth, it is the loaded quality factor that cannot be put back into (9) and (11) directly. When two SMA connectors are inserted into the cavity, even if they are loosely coupled, the external quality factor must be taken into account. The relationship among loaded $\left(Q_{\text {load }}\right)$, unloaded $\left(Q_{\text {unload }}\right)$, and external $\left(Q_{\text {ext }}\right)$ quality factors of the proposed cylindrical resonator can be expressed as

$$
\frac{1}{Q_{\text {load }}}=\frac{1}{Q_{\text {unload }}}+\frac{2}{Q_{\text {ext }}}
$$

where the last term refers to two identical SMA connectors. In practice, $Q_{\text {unload }}$ is found using eigenmode simulations in EDT. Besides, the scattering parameters are measured at the beginning for the cylindrical resonator with homogeneous conductor walls, and the quality factor is calculated. By putting both $Q_{\text {unload }}$ and $Q_{\text {load }}$ into (12), $Q_{\text {ext }}$ can be rewritten as

$$
Q_{\text {ext }}=\frac{2}{Q_{\text {load }}^{-1}-Q_{\text {unload }}^{-1}} .
$$

Once the external quality factor for the SMA connectors is determined, the value is kept constant and can be used for the following measurements. As a result, the quality factors involved in (9) and (11) can be calculated as

$$
Q=\frac{1}{Q_{\text {meas }}^{-1}-2 Q_{\text {ext }}^{-1}}
$$

where $Q_{\text {meas }}$ represents the measured quality factor of the proposed cylindrical resonator with specific top covers.

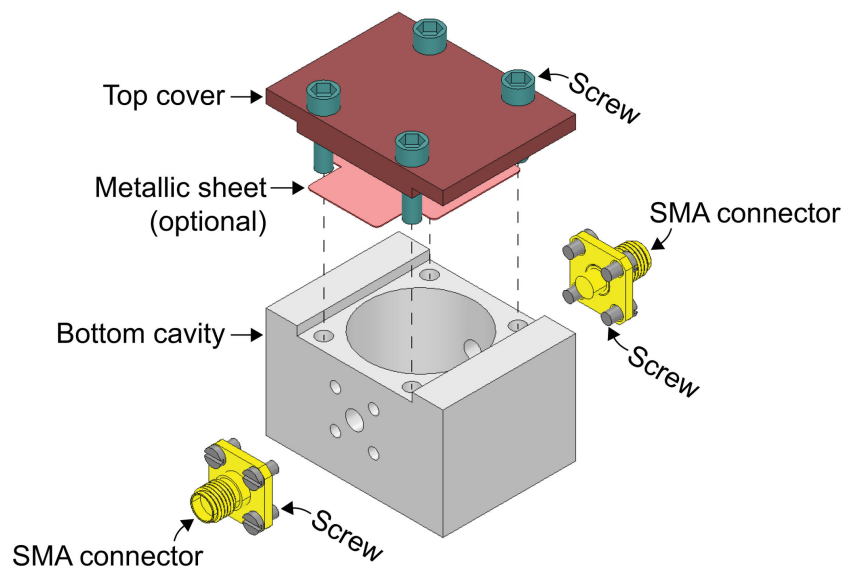

Fig. 5. Assembly structure of the proposed $\mathrm{TE}_{111}$ mode cylindrical resonator.

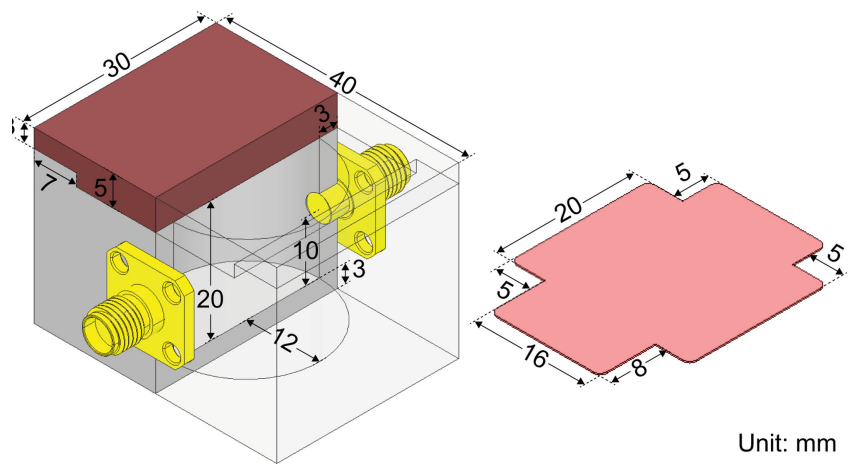

Fig. 6. Dimensions of the proposed $\mathrm{TE}_{111}$ mode cylindrical resonator and the metallic sheet.

\section{PROTOTYPE FABRICATION}

In order to validate the proposed cylindrical resonator as well as the method for characterizing the electrical conductivity, a prototype operating at $10.5 \mathrm{GHz}$ was fabricated. Fig. 5 demonstrates the assembly structure of the proposed $\mathrm{TE}_{111}$ mode cylindrical resonator. The resonator is divided into two parts, where the top cover is fixed to the bottom cavity using four screws. Two SMA connectors are inserted and cut along the side surface of the cylindrical resonator for a loosely coupling. In addition, for measuring the metallic sheets, the sample can be clamped between the top cover and the bottom cavity forming a sandwich structure. The bulge on the top cover and the recess on the bottom cavity ensure the alignment and contact of the metallic sheets.

The detailed dimensions of the proposed $\mathrm{TE}_{111}$ mode cylindrical resonator and the metallic sheet are illustrated in Fig. 6. The radius $(a)$ and the height $(d)$ of the cylindrical resonator are 12 and $20 \mathrm{~mm}$, respectively. The SMA connectors are located halfway between the top and bottom surfaces. The metallic sheets for characterizing the electrical conductivity must be large enough so that the top surface of the cylindrical resonator can be fully covered. Besides, the positions of the screws on the top cover need to be taken into account. As a consequence, the metallic sheets turn out to have a rectangular shape with corner cuts. 


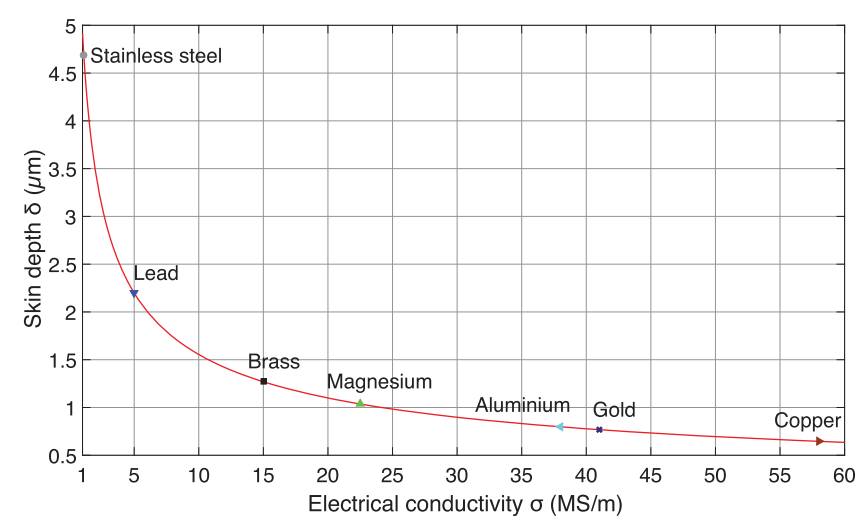

Fig. 7. Skin depth at $10.48 \mathrm{GHz}$ for different metallic materials $\left(\mu_{\mathrm{r}}=1\right)$ in terms of their typical values of the electrical conductivity.

Once the dimensions are determined, the resonant frequency $\left(f_{0}\right)$ of the proposed $\mathrm{TE}_{111}$ mode cylindrical resonator can be estimated using (3) as

$$
\begin{aligned}
f_{0} & =\frac{299792458}{2 \pi \sqrt{1 \cdot 1}} \sqrt{\left(\frac{1.841}{0.012}\right)^{2}+\left(\frac{\pi}{0.02}\right)^{2}} \\
& =10.48 \mathrm{GHz} .
\end{aligned}
$$

When measuring the electrical conductivity of the metallic sheets, the thickness of the samples should be at least twice of the skin depth at the resonant frequency. Otherwise, the measured electrical conductivity might be affected by the top cover material. The skin depth $(\delta)$ for nonmagnetic $\left(\mu_{\mathrm{r}}=1\right)$ materials can be written as

$$
\begin{aligned}
\delta & =\sqrt{\frac{2}{\omega \mu \sigma}}=\sqrt{\frac{1}{\pi f_{0} \mu_{0} \mu_{\mathrm{r}} \sigma}} \\
& =\sqrt{\frac{1}{\pi \cdot 10.48 \times 10^{9} \cdot 4 \pi \times 10^{-7} \cdot 1 \cdot \sigma}}
\end{aligned}
$$

where $\sigma$ represents the electrical conductivity of the material. The relationship between the skin depth and the electrical conductivity is presented in Fig. 7. The markers on the solid line indicate the calculated skin depths of the corresponding metallic materials using their typical values of the electrical conductivity [18], [19]. For the material with a higher electrical conductivity, a smaller skin depth is achieved. In this case, for example, a thin cladding layer outside the original metallic material with a thickness of several micrometers $(\mu \mathrm{m})$ should be enough to improve the electrical performance.

Fig. 8 demonstrates the fabricated cylindrical resonator. The prototype was fabricated using aluminum blocks by a milling process. Besides, extra brass and copper top covers were fabricated as references for comparing the characterized electrical conductivity of the metallic sheets.

The fabricated metallic sheets shown in Fig. 9 include material samples made by steel (S235), stainless steel (304), brass, aluminum, and copper. These samples were cut for fitting the cylindrical resonator, and their measured thicknesses are much more than the corresponding skin depth at the resonant frequency. In order to have a lower and identical surface roughness, the raw

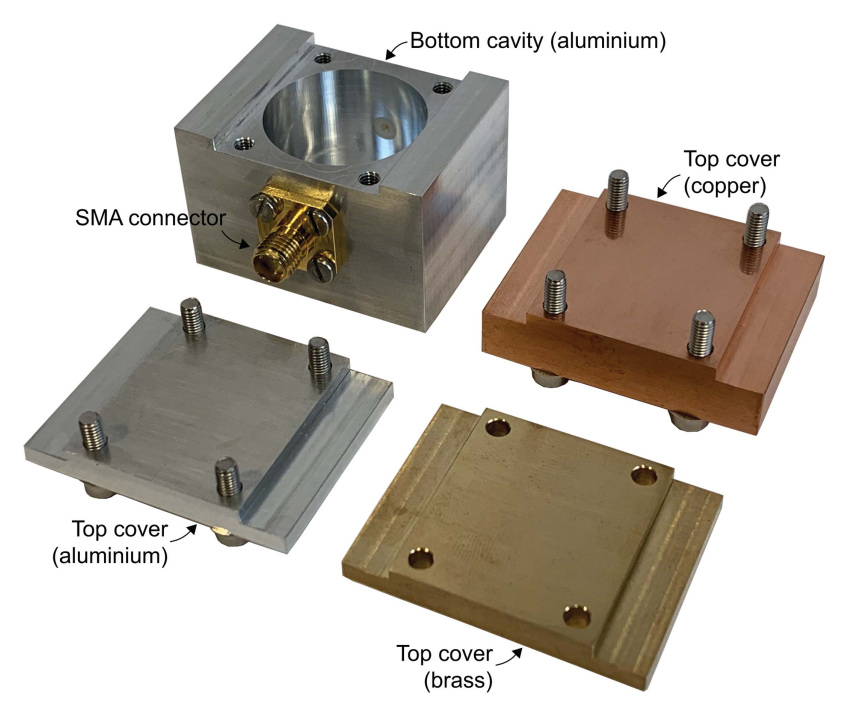

Fig. 8. Fabricated $\mathrm{TE}_{111}$ mode cylindrical resonator with aluminum, brass, and copper top covers.
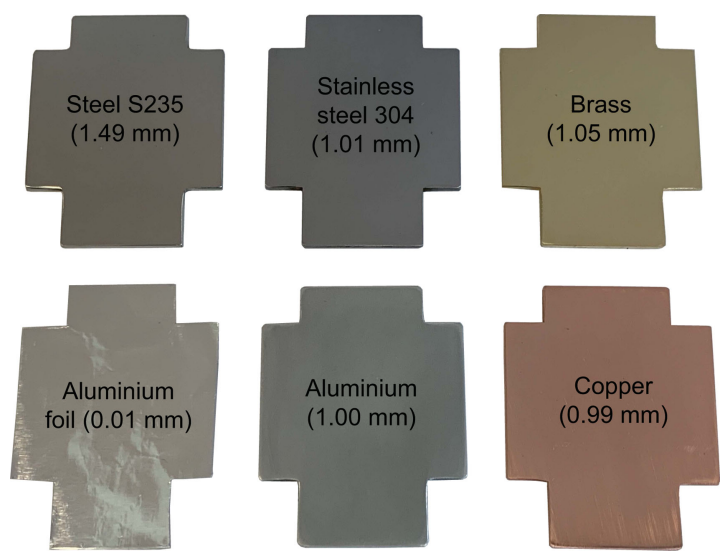

Fig. 9. Fabricated metallic sheet samples.

metallic sheets were polished by using an abrasive paper with a grit designation of P2000. As a supplement, a sample made by the aluminum foil used in the kitchen with a thickness of $0.01 \mathrm{~mm}$ is also included.

\section{EXPERIMENTAL RESULTS}

As shown in Fig. 10, the cylindrical resonator is connected to an Agilent E8361A vector network analyzer (VNA), which measures the scattering parameters of the device under test (DUT). In order to improve the accuracy, a two-port short-openload-through calibration is carried out prior to the measurements using an Agilent 85052D calibration kit, so that the effects of the cables as well as the connectors are calibrated out from the measured scattering parameters.

At the beginning, the external quality factor of the SMA connector $\left(Q_{\text {ext }}\right)$ needs to be found so that it can be used for the following measurements. The aluminum top cover is used, and the scattering parameters were measured. As shown in Fig. 11, the solid line represents the magnitude of the measured $S_{21}$. In addition, $f_{0}, f_{1}$, and $f_{2}$ correspond to the resonant 


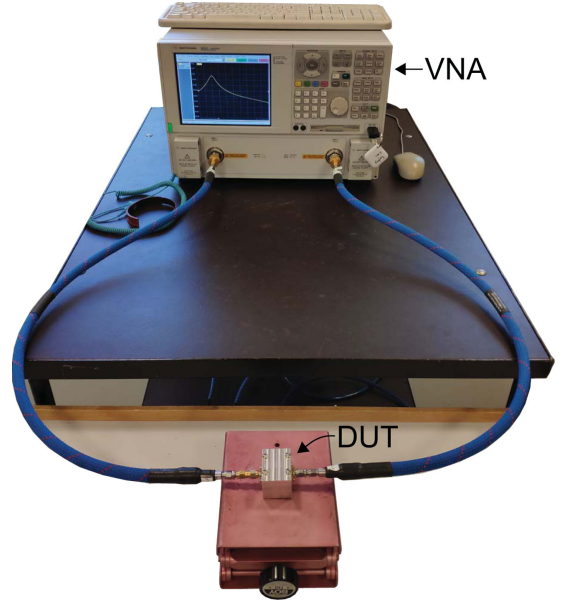

Fig. 10. Experimental setup for measuring scattering parameters of the DUT using a VNA.

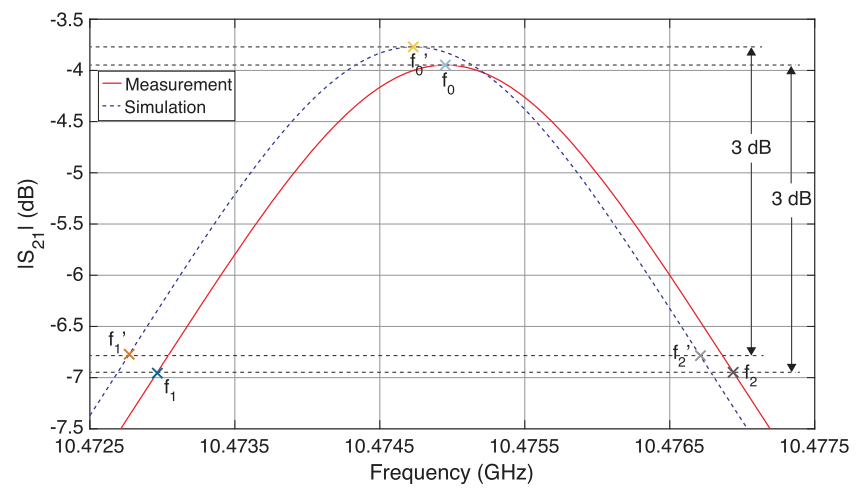

Fig. 11. Measured and simulated $\left|\mathrm{S}_{21}\right|$ of the proposed cylindrical resonator with the aluminum top cover for finding the loaded quality factor.

frequency and the lower and upper frequencies of the half-power bandwidth, respectively.

The loaded quality factor $\left(Q_{\text {load }}\right)$ is then calculated as

$$
\begin{aligned}
Q_{\text {load }} & =\frac{f_{0}}{f_{2}-f_{1}} \\
& =\frac{10474952000}{10476940000-10472964000} \\
& =2634.5 .
\end{aligned}
$$

Meanwhile, the unloaded quality factor $\left(Q_{\text {unload }}\right)$ is estimated using an eigenmode simulation in EDT. By taking into account the electrical conductivity and the surface roughness of the fabricated cylindrical resonator, a value of 7800 is achieved for $Q_{\text {unload. }}$. Thus, $Q_{\text {ext }}$ can be calculated by (13) as

$$
Q_{\text {ext }}=\frac{2}{\frac{1}{2634.5}-\frac{1}{7800}}=7956.5 .
$$

After that, the scattering parameters of the fabricated cylindrical resonator with different top covers and metallic sheets are measured so that the measured quality factors $\left(Q_{\text {meas }}\right)$ are calculated from the corresponding half-power bandwidth. Since the external quality factor of the SMA connector is found by (18) and kept constant, the quality factor $(Q)$ for each measurement can then be calculated by (14). Table I concludes the corresponding frequencies for the measured half-power bandwidth and the calculated quality factor.

During the characterization process, by using the calculated quality factors, the surface resistivity of aluminum is first derived by (11) and used as $R_{\mathrm{S} 1}$ putting back into (9). In this case, the surface resistivities of other top covers as well as metallic sheets can be derived by (9) afterward. At the last step, the surface resistivities are converted into the electrical conductivities by (10). The characterized electrical conductivities are also summarized in Table I and compared with other published results in the literature.

Among all tested material samples, the metallic sheet made by steel (S235) achieves the lowest electrical conductivity of $0.52 \mathrm{MS} / \mathrm{m}$. In addition, a similar performance can be observed for the metallic sheet made by stainless steel (304), which shows an electrical conductivity of $0.54 \mathrm{MS} / \mathrm{m}$. For the material samples made by brass, the characterized electrical conductivities of the metallic sheet and top cover show a difference of 5.3\%, which are 12.93 and $13.65 \mathrm{MS} / \mathrm{m}$, respectively. Three material samples are made by aluminum, including a metallic sheet, a top cover, and a foil. The characterized electrical conductivities are 22.91 and $21.90 \mathrm{MS} / \mathrm{m}$ for the metallic sheet and top cover, respectively. By contrast, due to a better surface roughness, the foil exhibits a higher electrical conductivity of $29.25 \mathrm{MS} / \mathrm{m}$. As for the metallic sheet and top cover made by copper, in comparison with other tested material samples, they achieve the highest electrical conductivities of 57.48 and $59.06 \mathrm{MS} / \mathrm{m}$, respectively.

When compared with the characterized electrical conductivities in [1] and [2], due to different characterization methods, frequencies, and material properties, a reasonable agreement has been achieved. For the method introduced in [1], a TE 011 mode cavity resonator is used for characterizing the electrical conductivity of metallic materials. The scattering parameters are measured using a VNA through two coupling loops on the top surface of the cavity. Besides, $Q_{\text {unload }}$ of the cavity resonator is calculated from the measured insertion loss and $Q_{\text {load, }}$, while the conductor loss of the cavity resonator is not taken into account separately. For circular waveguides, $\mathrm{TE}_{01}$ mode not only has a low attenuation at microwave frequencies but also results in simplified field distributions. However, $\mathrm{TE}_{01}$ mode is not the dominant mode of circular waveguides so that the proposed $\mathrm{TE}_{011}$ mode cavity resonator has larger dimensions. When operating at $8.4 \mathrm{GHz}$, the diameter of the tested material samples is around $100 \mathrm{~mm}$. By contrast, the proposed $\mathrm{TE}_{111}$ mode cylindrical resonator shown in Fig. 6 is more compact. When operating at $10.5 \mathrm{GHz}$, the length and the width of the tested material samples are 33 and $26 \mathrm{~mm}$, respectively. Furthermore, the diameter of the tested material samples can be reduced to $25 \mathrm{~mm}$ when necessary.

In [2], a tunable cavity resonator with a dielectric resonator and a quartz spacer is proposed for characterizing the electrical conductivity of metallic materials. The input impedance is measured through an SMA connector with a loop, which is coupled to the magnetic field in the cavity. By using a tuning plunger controller, the measurement is performed over a small range of 
TABLE I

Measured Frequencies, Calculated Quality Factor, and Characterized Electrical Conductivity at 10.5 GHz

\begin{tabular}{|c|c|c|c|c|c|c|c|c|c|c|}
\hline Material & Type & $\begin{array}{c}f_{0} \\
(\mathrm{GHz})\end{array}$ & $\begin{array}{c}f_{1} \\
(\mathrm{GHz})\end{array}$ & $\begin{array}{c}f_{2} \\
(\mathrm{GHz})\end{array}$ & $Q_{\text {meas }}$ & $Q^{*}$ & $\begin{array}{c}\sigma \\
(\mathrm{MS} / \mathrm{m})\end{array}$ & $\begin{array}{c}{[1]^{\star}} \\
(\mathrm{MS} / \mathrm{m})\end{array}$ & $\begin{array}{c}{[2]^{\diamond}} \\
(\mathrm{MS} / \mathrm{m})\end{array}$ & $\begin{array}{c}\text { Typ. }^{\dagger} \\
\text { (MS/m) }\end{array}$ \\
\hline \multirow{2}{*}{ Steel } & sheet, S235 grade & 10.47887560 & 10.47610680 & 10.48169800 & 1892.3 & 3609.0 & 0.52 & \multirow{2}{*}{ na } & \multirow{2}{*}{ na } & \multirow{2}{*}{1.1} \\
\hline & sheet, 304 stainless & 10.47511340 & 10.47236750 & 10.47790310 & 1907.4 & 3664.3 & 0.54 & & & \\
\hline Brass & sheet & 10.47617600 & 10.47414025 & 10.47820425 & 2573.1 & 7284.6 & 12.93 & 13.2 & na & 15.0 \\
\hline \multirow{3}{*}{ Aluminium } & sheet & 10.47617310 & 10.47418290 & 10.47816570 & 2631.9 & 7777.2 & 22.91 & \multirow{3}{*}{23.1} & \multirow{3}{*}{21.3} & \multirow{3}{*}{38.2} \\
\hline & top cover & 10.47511100 & 10.47312230 & 10.47710870 & 2627.7 & 7740.4 & 21.90 & & & \\
\hline & foil & 10.48857320 & 10.48659720 & 10.49056400 & 2654.0 & 7973.0 & 29.25 & & & \\
\hline Copper & sheet & 10.47647500 & 10.47453550 & 10.47840900 & 2700.8 & 8411.1 & 57.48 & 46.8 & 55.4 & 58.1 \\
\hline
\end{tabular}

$* Q_{\text {ext }}=7956.5$ is used for calculations.

* In [1], the measurements were carried out at $8.4 \mathrm{GHz}$

${ }^{\diamond}$ In [2], the measurements were carried out at $3.5 \mathrm{GHz}$.

${ }^{\dagger}$ The conductivities reported in [18] and [19] are used as the typical values.

frequencies. However, it has a complex structure, and $Q_{\text {unload }}$ of the cavity resonator is obtained using a finite integration algorithm. The quartz spacer is also assumed to be lossless, and an estimated value is used as the electrical conductivity of the cavity resonator.

The electrical conductivies reported in [18] and [19] are referenced as the typical values in Table I. Since the measurements are carried out at dc using high-purity materials, some of the typical values are much higher than the characterized electrical conductivities. In particular, for aluminum, including the foil sample, the characterized electrical conductivities range from 21.3 to $29.25 \mathrm{MS} / \mathrm{m}$, while the typical value is $38.2 \mathrm{MS} / \mathrm{m}$. At microwave frequencies, the electrical conductivities are mainly affected by skin depth, impurities, oxidization, and surface roughness. As a result, for critical applications, instead of directly using the typical values, the electrical conductivities of the customized materials or samples are usually characterized at the operating frequency.

\section{ACCURACY AND UNCERTAINTY}

For the proposed method, as an essential parameter for extracting the external quality factor $\left(Q_{\text {ext }}\right)$, the unloaded quality factor ( $\left.Q_{\text {unload }}\right)$ of the cylindrical resonator needs to be determined at the beginning, and its accuracy also affects the following characterization process. However, the estimation of $Q_{\text {unload }}$ is sorely based on an eigenmode simulation in EDT. Under this circumstance, the simulation accuracy and the uncertainty of the characterized electrical conductivities need to be taken into consideration. In order to improve the accuracy of the eigenmode simulation, aluminum is assigned as the material of the cylindrical resonator, while the roughness of the inner surfaces is set to be around $0.35 \mu \mathrm{m}$. Though the actual electrical conductivity and surface roughness of the fabricated cylindrical resonator might be slightly different from the values used for the eigenmode simulation, it represents an overall and comprehensive effect, which models the imperfections of the cylindrical resonator including fabrication tolerance, surface roughness, and structural discontinuity between the top cover and the bottom cavity.

When the same material and surface roughness settings are used for simulating the cylindrical resonator with SMA connectors, the accuracy can be evaluated by comparing the loaded quality factors that are calculated from the measured and simulated scattering parameters. The dashed line in Fig. 11 represents the magnitude of the simulated $S_{21}$. The simulated loaded quality factor $\left(Q_{\text {load }}^{\prime}\right)$ is then calculated as

$$
\begin{aligned}
Q_{\text {load }}^{\prime} & =\frac{f_{0}^{\prime}}{f_{2}^{\prime}-f_{1}^{\prime}} \\
& =\frac{10474740000}{10476702600-10472772300} \\
& =2665.1
\end{aligned}
$$

where $f_{0}^{\prime}, f_{1}^{\prime}$, and $f_{2}^{\prime}$ refer to the simulated resonant frequency and lower and upper frequencies of the half-power bandwidth, respectively. In comparison with the measured loaded quality factor $\left(Q_{\text {load }}\right)$ calculated by (17), a good agreement is achieved. Since the difference between $Q_{\text {load }}$ and $Q_{\text {load }}^{\prime}$ is kept less than $1.2 \%$, the material and surface roughness settings used in the simulations are validated. As a result, $Q_{\text {unload }}$ with a simulated value of 7800 is used for the proposed method.

The characterized electrical conductivities listed in Table I are calculated from the measured scattering parameters, while $Q_{\text {ext }}$ is also involved and extracted using $Q_{\text {unload }}$ of the cylindrical resonator. Thus, the uncertainty caused by the estimation of $Q_{\text {unload }}$ is transferred to the characterized electrical conductivities. However, the potential effect and the corresponding relationship are not straightforward. Fig. 12 illustrates the variations of the characterized electrical conductivity in terms of $Q_{\text {unload }}$ for different material samples. When $Q_{\text {unload }}$ changes within 


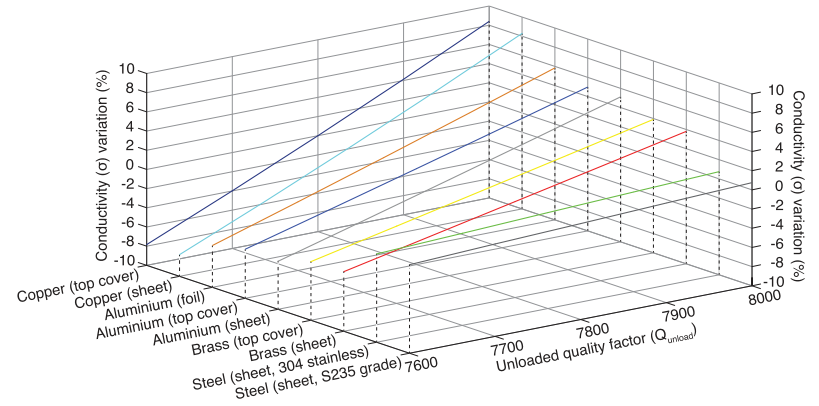

Fig. 12. Variations of the characterized electrical conductivity due to the uncertainty of the unloaded quality factor.

TABLE II

UNCERTAINTY OF THE CHARACTERIZED ELECTRICAL CONDUCTIVITY

\begin{tabular}{lll}
\hline Material & Type & Uncertainty* \\
\hline Steel & sheet, S235 grade & $+0.4 \% /-0.3 \%$ \\
& sheet, 304 stainless & $+0.3 \% /-0.4 \%$ \\
\hline Brass & sheet & $+1.8 \% /-1.8 \%$ \\
& top cover & $+1.8 \% /-1.8 \%$ \\
\hline \multirow{3}{*}{ Aluminium } & sheet & $+2.4 \% /-2.4 \%$ \\
& top cover & $+2.3 \% /-2.3 \%$ \\
& foil & $+2.7 \% /-2.7 \%$ \\
Copper & sheet & $+3.8 \% /-3.7 \%$ \\
& top cover & $+3.9 \% /-3.7 \%$ \\
\hline
\end{tabular}

${ }^{*}$ The uncertainty of $Q_{\text {unload }}$ is $\pm 1.2 \%$.

the range of $7800 \pm 200$, it corresponds to an uncertainty of $\pm 2.6 \%$. In this case, the material samples with higher electrical conductivities are more sensitive and exhibit larger variations.

Table II lists the derived uncertainties of the characterized electrical conductivities for different material samples when $Q_{\text {unload }}$ has an uncertainty of $\pm 1.2 \%$. With the highest electrical conductivity among all tested materials, copper achieves an uncertainty of $\pm 3.9 \%$, while it is only $\pm 0.4 \%$ for steel. In addition, the uncertainties for aluminum and brass are $\pm 2.7 \%$ and $\pm 1.8 \%$, respectively.

Except for the estimation of $Q_{\text {unload }}$, several assumptions are involved in the characterization process. The proposed cylindrical resonator is filled with air in reality, while vacuum is used for calculations. Both the relative permeability $\left(\mu_{\mathrm{r}}\right)$ and the relative permittivity $\left(\varepsilon_{\mathrm{r}}\right)$ are assumed to be 1 . The dissipation factor $(\tan \delta)$ is assumed to be 0 at the operating frequency. Since the measurements are carried out in the laboratory at room temperature, the influence caused by the vacuum assumption can be neglected. However, for extreme situations such as high humidity environment, the vacuum assumption becomes inappropriate, and the variations of the properties have to be taken into account.

The electric field $(E)$ and magnetic field $(H)$ components of the $\mathrm{TE}_{n m l}$ mode cylindrical resonator introduced in (1) are derived using Maxwell's equations with lossless assumption. In general, the propagation constant $(\gamma)$ is a complex number consisting of both the attenuation constant $(\alpha)$ and the phase constant $(\beta)$. However, due to low conductor loss of the metallic materials and short physical length of the cavity, $\alpha$ is much smaller than $\beta$ for the proposed cylindrical resonator. Thus, $\alpha$ can be neglected, while $\beta$ is used as the propagation constant for calculations. For materials with high dielectric loss or conductor loss, the assumption for Maxwell's equations is not valid since $\alpha$ becomes comparable with $\beta$. In this case, the field components of the cylindrical resonator have to be expressed in a more complex format.

\section{CONCLUSION}

In this article, a novel method for characterizing the electrical conductivity of metallic materials using a cylindrical resonator has been presented. The average stored energy in the cylindrical resonator and the power loss due to nonperfect metallic materials are first derived using Maxwell's equations. The quality factor is then expressed in terms of the surface resistivities of the top, bottom, and side surfaces. Under this circumstance, once the quality factor is measured and the surface resistivity of the bottom and side surfaces are known, the electrical conductivity of the top surface can be characterized at the resonant frequency. The proposed method provides an easy and realistic way of measuring the electrical conductivity of metallic materials at microwave frequencies. Due to skin effect, the method is suitable for testing not only conventional top covers and metallic sheets but also thin material samples such as foil and cladding layers. As a prototype, a $\mathrm{TE}_{111}$ mode cylindrical resonator operating at $10.5 \mathrm{GHz}$ has been fabricated using aluminum blocks by a milling process. In order to validate the method, three top covers and six metallic sheets have been fabricated and tested. The characterized electrical conductivities are compared with other published results in the literature, and a reasonable agreement has been achieved. The uncertainties of the characterized electrical conductivities are also discussed. Furthermore, by adjusting the resonant frequency of the cylindrical resonator, the proposed method can be potentially used for characterizing electrical conductivities at millimeter-wave and terahertz frequencies.

\section{ACKNOWLEDGMENT}

The authors would like to thank M. Nielsen, J. Lauridsen, and J. N. Mortensen at the Department of Electrical Engineering, Technical University of Denmark, for assisting the fabrication processes.

\section{REFERENCES}

[1] T. Y. Otoshi and M. M. Franco "The electrical conductivities of steel and other candidate materials for shrouds in a beam-waveguide antenna system," IEEE Trans. Instrum. Meas., vol. 45, no. 1, pp. 77-83, Feb. 1996.

[2] D. Kajfez and A. Gundavajhala, "Measurement of material properties with a tunable resonant cavity," Electron. Lett., vol. 29, no. 22, pp. 1936-1937, Oct. 1993.

[3] W. Frey, "Microwave measurement of surface conductivity and permittivity of thin layers in an $E_{010}$ resonator," Electron. Lett., vol. 8, no. 19, pp. 486-488, Sep. 1972.

[4] Z. Wang et al., "Characterization of thin metal films using terahertz spectroscopy," IEEE Trans. THz Sci. Technol., vol. 8, no. 2, pp. 161-164, Mar. 2018. 
[5] M. P. Kirley and J. H. Booske, "Terahertz conductivity of copper surfaces," IEEE Trans. THz Sci. Technol., vol. 5, no. 6, pp. 1012-1020, Nov. 2015.

[6] J. Chung, N. K. Nahar, L. Zhang, Y. Bayram, K. Sertel, and J. L. Volakis, "Broadband radio frequency conductivity measurement technique for engineered composites," IET Microw. Antennas Propag., vol. 6, no. 4, pp. 371-376, Mar. 2012.

[7] A. Stadler, M. Albach, and A. Lindner, "A practical method to measure electrical AC conductivity of MnZn ferrites using conventional toroids," IEEE Trans. Magn., vol. 46, no. 2, pp. 678-681, Feb. 2010.

[8] M. A. Hindy, "Optimized method for obtaining permittivity and conductivity profiles of microwave materials," IEEE Trans. Microw. Theory Techn., vol. 37, no. 5, pp. 922-925, May 1989.

[9] Z. Wang and Y. Yu, "Thickness and conductivity measurement of multilayered electricity-conducting coating by pulsed eddy current technique: Experimental investigation," IEEE Trans. Instrum. Meas., vol. 68, no. 9, pp. 3166-3172, Sep. 2019.

[10] C. Wang, M. Fan, B. Cao, B. Ye, and W. Li, "Novel noncontact eddy current measurement of electrical conductivity," IEEE Sens. J., vol. 18, no. 22, pp. 9352-9359, Nov. 2018.

[11] E. Pinotti and E. Puppin, "Simple lock-in technique for thickness measurement of metallic plates," IEEE Trans. Instrum. Meas., vol. 63, no. 2, pp. 479-484, Feb. 2014.

[12] A. Girgis and A. Bastawros, "Nondestructive eddy current testing for the measurement of conductivity and surface buckling of metallic sheets," IEEE Trans. Instrum. Meas., IM- 35, no. 4, pp. 619-623, Dec. 1986.

[13] J. Krupka, J. Breeze, A. Centeno, N. Alford, T. Claussen, and L. Jensen, "Measurements of permittivity, dielectric loss tangent, and resistivity of float-zone silicon at microwave frequencies," IEEE Trans. Microw. Theory Techn., vol. 54, no. 11, pp. 3995-4001, Nov. 2006.

[14] E. Silva, N. Pompeo, K. Torokhtii, and S. Sarti, "Wideband surface impedance measurements in superconducting films," IEEE Trans. Instrum. Meas., vol. 65, no. 5, pp. 1120-1129, May 2016.

[15] Y. Nonaka, H. Nakane, T. Maeda, and K. Hasuike, "Simultaneous measurement of the resistivity and permeability of a film sample with a double coil," IEEE Trans. Instrum. Meas., vol. 44, no. 3, pp. 679-682, Jun. 1995.

[16] K. Kikuchi, "Measurement of the surface resistance of a metallic thin film at a wavelength of $10.6 \mu \mathrm{m}$," IEEE Trans. Instrum. Meas., vol. 39, no. 2, pp. 395-398, Apr. 1990.

[17] J. R. S. Avila, K. Y. How, M. Lu, and W. Yin, "A novel dual modality sensor with sensitivities to permittivity, conductivity, and permeability," IEEE Sens. J., vol. 18, no. 1, pp. 356-362, Jan. 2018

[18] D. M. Pozar, Microwave Engineering. Hoboken, NJ, USA: Wiley, 2012.

[19] P. A. Rizzi, Microwave Engineering: Passive Circuits. Englewood Cliffs, NJ, USA: Prentice-Hall, 1988.

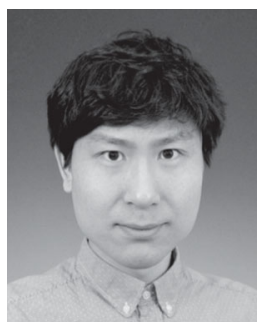

Yunfeng Dong (Member, IEEE) received the B.Sc. degree in electronic information engineering from the Beijing University of Technology, Beijing, China, in 2012, and the M.Sc. and Ph.D. degrees in electrical engineering from the Technical University of Denmark (DTU), Kongens Lyngby, Denmark, in 2014 and 2019 , respectively.

From 2011 to 2012, he was an Exchange Student with the South-Eastern Finland University of Applied Sciences, Mikkeli, Finland. In 2014, he was a Research Assistant with the Electromagnetic Systems Group, DTU, where he is currently a Postdoctoral Researcher. In 2017, he was a Visiting Scholar with the Terahertz Devices and Systems Group, Technische Universität Darmstadt, Darmstadt, Germany. His research interests include developing innovative strategies that are suitable for hybrid integration and packaging at millimeter-wave and terahertz frequencies.

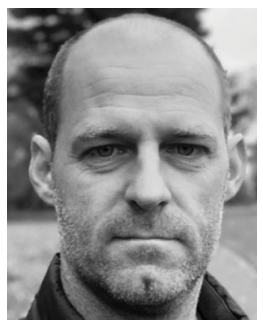

Morten Stendahl Jellesen received the M.Sc. and Ph.D. degrees in corrosion and surface technology from the Technical University of Denmark (DTU), Kongens Lyngby, Denmark, in 2004 and 2007, respectively.

He is currently a Senior Scientist with the Section of Materials and Surface Engineering, Department of Mechanical Engineering, DTU. Since 2007, he has been associated with the Centre for Electronic Corrosion projects. His research interests include soldering, surface plating, contamination, corrosion, and climatic reliability of electronics.

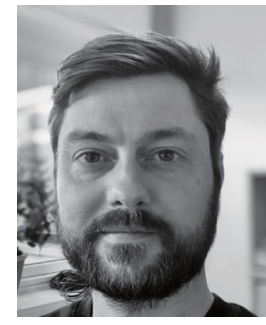

Rune Juul Christiansen received the M.Sc. degree in biomaterials from Aarhus University, Aarhus, Denmark, in 2010, and the Ph.D. degree in corrosion and surface technology from the Technical University of Denmark, Kongens Lyngby, Denmark, in 2016.

Since 2016, he has been a Postdoctoral Researcher with AH Metal Solutions A/S, Allerød, Denmark, where his work involves material science related to formability and corrosion of metal alloys. His research interests include electrochemical analysis of metal alloys used in biomedical applications, biocompatibility of metal alloys, deformation-induced corrosion in austenitic steels, and electrophysiology.

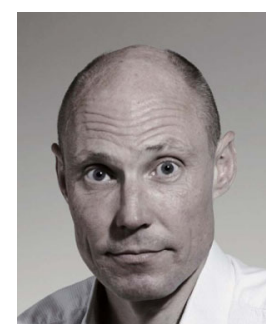

Jesper Høvelskov received the B.S. degree in economy and business administration from the Copenhagen Business School (CBS), Frederiksberg, Denmark, in 1993.

Since 2004, he has been the owner and CEO of AH Metal Solutions A/S, Allerød, Denmark. Previously, he was the CEO of ScanCastor A/S, Jyllinge, Denmark, and held various positions including Quality and Project Manager in the family company, $\mathrm{AH}$ Metal Solutions A/S. He has led countless development projects with industry leading companies of creating high-precision metal components. AH Metal Solutions A/S is currently producing millions of high-precision metal components annually. Under his management, AH Metal Solutions A/S has grown to be a highly renowned company engaging in the state-of-the-art research aiming at innovative product development.

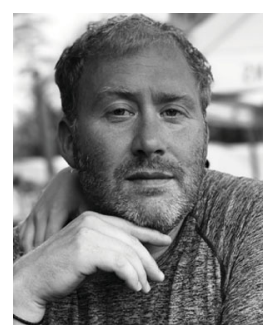

Jörgen Sundgren received eduacation in electrical and programming for Programmable Logic Controller (PLC) and Business Economy, in 1995 and 1998, respectively.

$\mathrm{He}$ is currently a part owner and CEO of Lysekil Plating Technology (LPTech) AB, Lysekil, Sweden. Since 2015, he has been a Technical Advisor with Elplatek/GalvaNord A/S, Espergæred, Denmark. He has been working with surface treatment since he was a teenager both in family business and as an employee. He worked for 17 years with one of the largest surface treatment companies in Scandinavia as a Technical Manager, Production Manager, and part owner. During this period, the company grew from a small family business to one of the largest, which offers about 90 different types of surface treatment. Now, the LPTech AB is growing as well and was noticed by the Swedish Agency for Economic and Regional Growth (Tillväxtverket) in 2017 due to the doubled yearly sales.

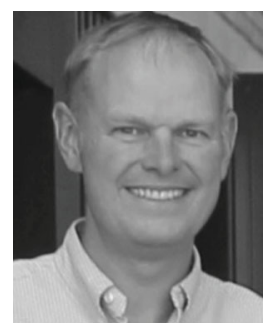

Kaj Bjarne Jakobsen received the B.Sc.E.E. and M.Sc.E.E. degrees in antennas and optical communication from the Technical University of Denmark (DTU), Kongens Lyngby, Denmark, in 1985 and 1986, respectively, the Ph.D. degree in electrical engineering from the University of Dayton, Dayton, $\mathrm{OH}$ USA, in 1989, and the H.D. degree in organization and management from the Copenhagen Business School, Frederiksberg, Denmark, in 2000.

From 1986 to 1989 , he was a Fulbright Scholar with the Department of Electrical Engineering, University of Dayton. Since 1990, he has been an Associate Professor with the Department of Electrical Engineering, DTU. His current research interests include body-centric wireless networks, wireless body area networks, and body sensor networks.

Dr. Jakobsen was a recipient of the NCR Stakeholder Award, Ohio, USA, in 1989, and the Appointed Teacher of the Year at DTU in 1994. 